\title{
Design of Stepping Motor control system based on LabVIEW
}

\author{
Yuan WANG ${ }^{1,2, a}$, Li Ping HUANG ${ }^{3, b}$,Jia Ning WANG ${ }^{1, c}$ \\ ${ }^{1}$ Institute of Science and Technology, Sanya University, Sanya, Hainan, China \\ ${ }^{2}$ Institute of Maritime Communication, Sanya University, Sanya, Hainan, China \\ ${ }^{3}$ Sanya No.1 Middle School, Sanya, Hainan, China \\ awangyuan0155@163.com, b564461732@qq.com, ‘495251263@qq.com
}

\begin{abstract}
Keywords: LabVIEW, Stepping Motor, NI ELVIS II Platform, Virtual Instrument
Abstract. By design the program and control the corresponding hardware devices, to design a Stepping motor control system to control the rotation of the stepping motor. The software part of this system is programmed based on LabVIEW, and communicates with the ELVIS II platform via USB port of the computer, so as to carry out a series of control on stepping motor. The hardware part consists of four parts, including the computer, the NI ELVIS II platform, the drive and the stepping motor. Through the LabVIEW program in the computer input the rotation angle. The rotation angle information is transmitted from the computer hardware interface to the NI ELVIS II platform and generates corresponding electrical signal. The electrical signal is transmitted to the driver and drives the Stepping motor to perform the corresponding rotation angle and control the rotation direction. The system test shows that the rotation angle is larger, the actual rotation error is smaller, and the frequency coefficient of stepping motor is larger, the actual rotation error is smaller. In addition to controlling the stepping motor, this system could be used in the production line and machine processing operation control.
\end{abstract}

\section{Introduction}

In the past two decades, with the development of digital technology and the improvement of stepping motor technology, stepping motor has played a significant role in the digital manufacturing era [1]. Especially in high precision device processing, high precision instrument control and automatic production process stepping motor play a vital role. The regulation of direction and speed of stepping motor is a milestone in industrial development, and its essence is to control the speed, deceleration, rotation angle and direction of the motor [2]. The principle of stepping motor is to convert the electrical pulse signal into the angular displacement or the line displacement actuator [3]. It can be seen as a synchronous motor running in a special way. Is widely known as stepping motor, because every time receives a pulse, stepping motor step, only the number of pulse also naturally become the main factors that control of stepping motor. Stepping motor has a large proportion in the mechanical market. Its largest application is in the manufacture of CNC milling machine. Since the stepping motor does not need A/D conversion, it can convert the digital pulse signal directly into angular displacement, which is considered to be the ideal numerical control milling machine implementation element [4]. And with the rapid development of science and technology, stepping motor can autonomously control an independent system and become an indispensable executive component.

As the leader of the virtual instrument, National Instruments (NI) company has made great contributions to hardware and software. LabVIEW is a development environment developed by NI company. LabVIEW and other computer language is a significant difference between other computer language is based on text language code, and the use of LabVIEW is a graphical language program, the program is in the form of block diagram. LabVIEW software is the core of designing virtual instrument, and it is also an ideal choice for developing measurement or control system.

This system is to use the LabVIEW software to control the stepping motor reversing, rotation angle acceleration and deceleration, and according to the needs of users to accurately fixed rotation angle, in order to achieve good control effect. 


\section{Stepping Motor Control System Design Scheme}

The overall design scheme of the stepping motor control system is shown in Fig. 1, which is divided into four parts. The first part is the computer, which is used to write the LabVIEW program, which is controlled by the program and sends out the pulse number instruction, which passes through the USB port to the NI ELVIS II platform. The second part is the ELVIS II platform developed by NI company, which contains a variety of commonly used laboratory instruments, equivalent to a small and versatile integrated instrument. Use the NI ELVIS II platform to generate directional control signals and continuously generate pulse signals. The third part is the drive that matches the stepping motor, which is composed of pulse generator control unit, power drive unit and protective power supply. The drive works by converting the received pulse signal into the angular displacement of the stepping motor. The impulse signal that converts the drive is considered as a small special pulse signal, and only when the pulse signal of this type is received, the stepping motor will turn. The fourth part is stepping motor, which is different from other $\mathrm{dc}$ or ac motor. The stepping motor could not work directly under normal conditions. The stepping motor can be controlled by controlling the signals emitted by the NI ELVIS II platform.

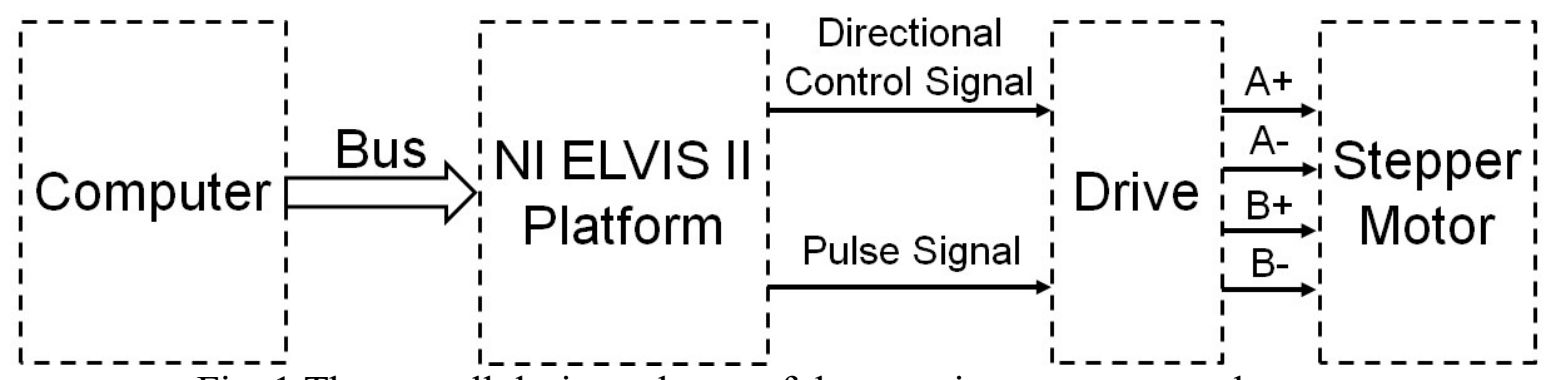

Fig. 1 The overall design scheme of the stepping motor control system

\section{Stepping Motor Control principle}

Speed Control Principle. The stepping motor is mainly used to give an electric signal to the corresponding windings, and the motor will run in a certain direction. And every time an electric signal motor is changed, the motor will run continuously if the response electrical signal is given continuously. If change the frequency of the electrical signal, will get a different speed. The stepping motor can be adjusted by changing the frequency of the output pulse of the function signal generator.

Steering Control Principle. The control of the stepping motor rotation direction is the control of the step motor's forward and backward rotation, which can change the rotation direction of the stepping motor by changing the power sequence [5]. Suppose that the switching power of the stepping motor is A-AB-B-BA, then the stepping motor is reversed when the order is BA-B-AB-A [6]. If there is a stepping motor drive matching it, the direction depends only on the high or low level of the direction the controller sends out.

Angle Control Principle. There are many ways to control the rotation angle of stepping motor. This paper adopts the method of timing. The stepping motor turns one circle of 200 steps, i.e., 1 step is $1.8^{\circ}$. According to the subdivision principle of the drive, if the frequency parameter is 1 , the minimum step angle is $1.8 / 1=1.8^{\circ}$. If the frequency parameter is 16 , the minimum step angle is $1.8 / 16=0.1125^{\circ}$, and so on. If want to go to the desired angle of the user, just need to figure out the time need to move the steps.

\section{Hardware Part of the Stepping Motor Control System}

This paper use the NI ELVIS II platform to control the stepping motor, which is actually programmed by LabVIEW to generate pulses through the virtual instrument platform indirectly controlling the drive. Stepping motor adopts 42BYGH34 two-phase four-wire stepping motor, and the drive also elects its matching TB6600 drive. 
Computer. The computer needs to install the LabVIEW software developed by NI company, the virtual instrument platform driver and the NI-DAQmx data collection driver. The computer is connected to the NI ELIVIS II virtual instrument platform by bus.

NI ELIVIS II Virtual Instrument Platform. NI ELVIS II platform is developed by NI company compact laboratory instrument platform, platform contains 12 kinds of commonly used laboratory instrument, including oscilloscope, digital multimeter, function generator, variable voltage source, potter analyzer, etc. [7]. In this system, the function of the platform is to convert the information of the LabVIEW program in the computer into the corresponding electrical signal, so as to control the stepping motor.

Stepping Motor. The stepping motor used in this paper is 42BYGH34 two-phase four-wire stepping motor. This type of stepping motor is one of the superior models in two-phase stepping motor. The step angle of the stepping motor is set at the factory time. In the case of no reduction gear, for an impulse signal, the mechanical angle of the rotor is the step angle [5]. For 42BYGH34 stepping motor, the frequency parameter to 1 , the minimum interval angle is $1.8^{\circ}$.

Drive. The stepping motor is different from an ordinary motor is that it needs a drive to work. When an impulse phase current is entered into the winding, the stepping motor rotor turns a set step angle. The driver model adopted in this design is TB6600. This drive is specially designed to drive the two-phase stepping motor, which can realize positive and reverse control. The 8 subdivision control by 3 bit dial switch of S1, S2 and S3, and 6 current control through 3 bit dial switch of S4, S5 and S6.

The hardware part of the stepping motor control system is shown in Fig. 2.

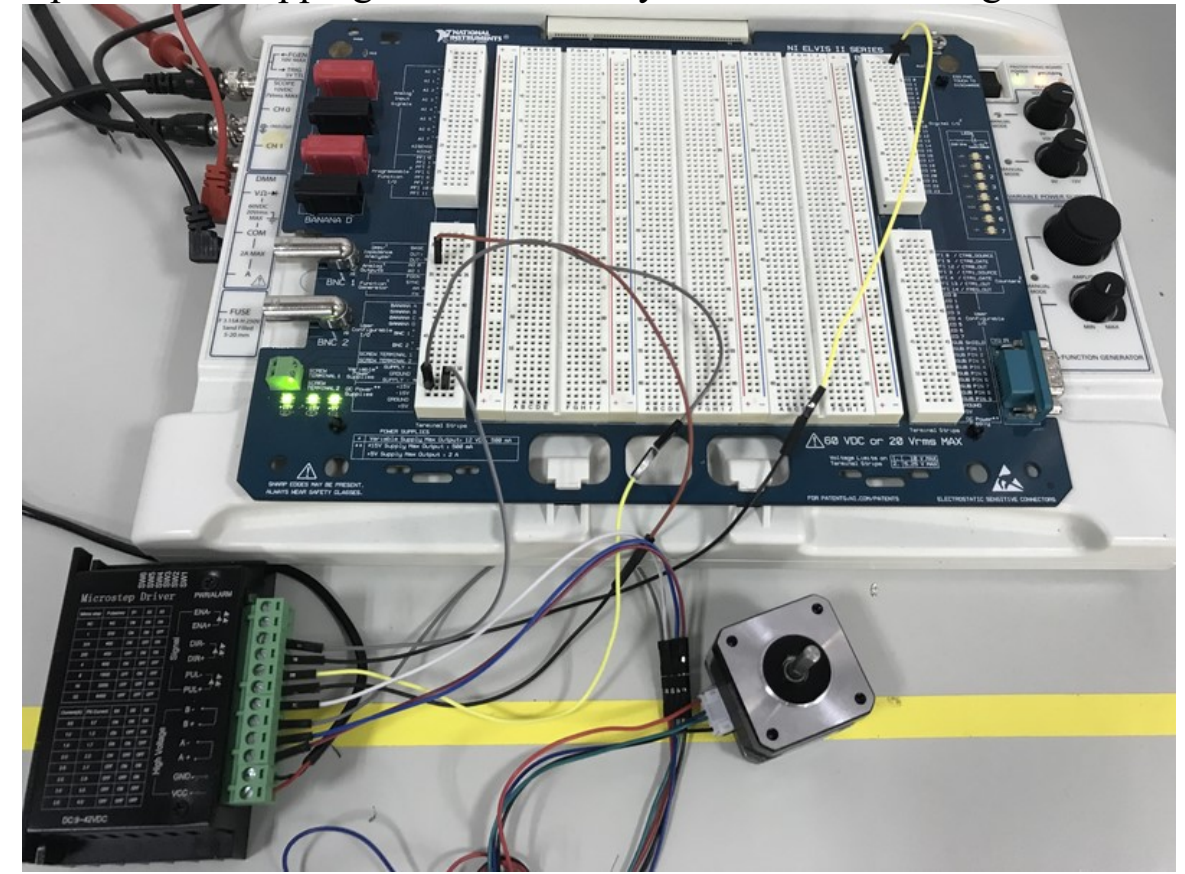

Fig.2 The hardware part of the stepping motor control system

\section{Software Part of the Stepping Motor Control System}

This procedure control principle is to use the function of the frequency of square wave signal generator to generate the user demand, if don't have any manipulation of the frequency, then the stepping electric opportunity continues to turn, and the speed is proportional to the user given frequency. The time required is calculated by multiplying the square wave frequency and the step number of the stepping motor, and this time is used to control the angle of the stepping motor turning. The positive end of the direction signal of the stepping motor is connected to the digital data acquisition port, and it is assigned to a Boolean variable. The positive and negative spin of the motor can be realized by changing the positive and negative of the Boolean variable. The program block diagram and front panel of the LabVIEW program is shown in Fig. 3 and Fig. 4. 


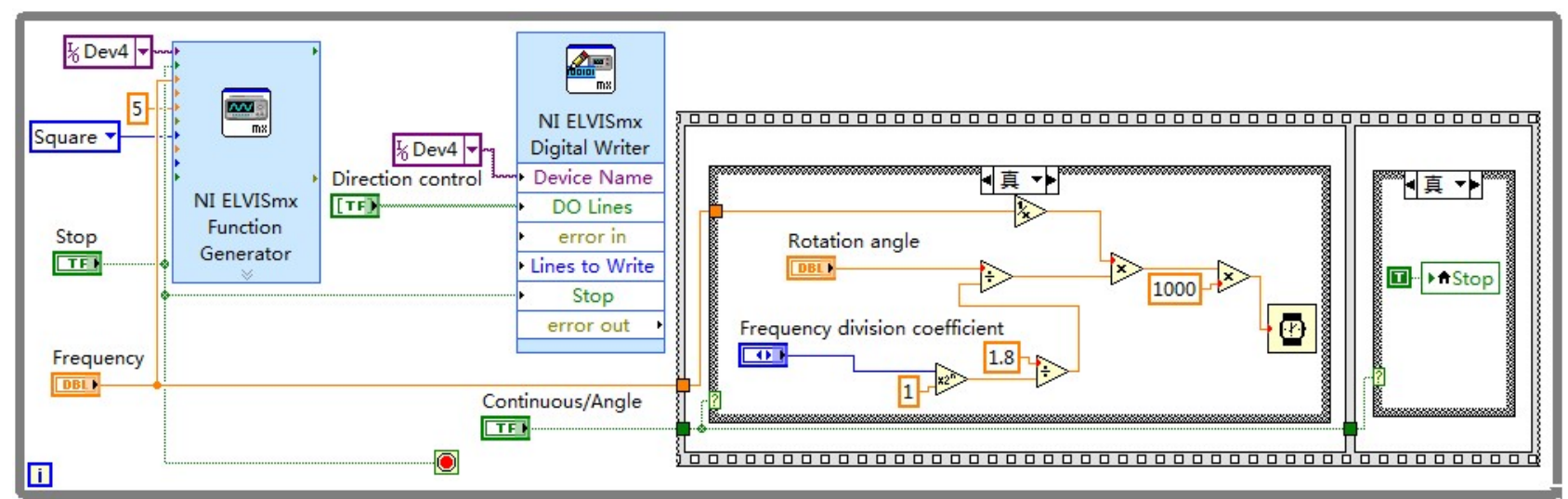

Fig. 3 The program block diagram of the LabVIEW program

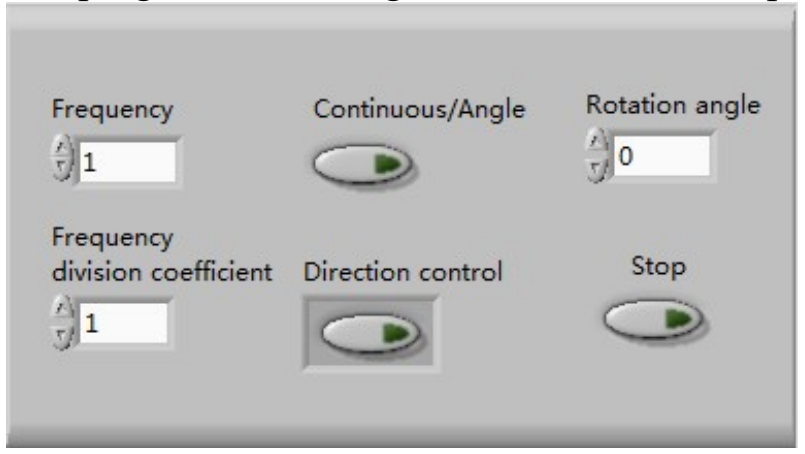

Fig. 4 The front panel of the LabVIEW program

\section{Test Results}

According to the hardware part and software part, build the stepping motor control system. On this basis, to analyze the rotation angle error of stepping motor at 16 minutes frequency, the test results are shown in the Table 1. At the same time, analyze the rotation angle error of stepping motor under different frequency division parameters, the test results are shown in the Table 2.

The system test results show that the rotation angle is larger, the actual rotation error is smaller, and the frequency coefficient of stepping motor is larger, the actual rotation error is smaller.

Table 1 Analyze the rotation angle error of stepping motor at 16 minutes frequency

\begin{tabular}{cccc} 
Rotation angle $\left[^{\circ}\right]$ & Actual rotation angle $\left[{ }^{\circ}\right]$ & Error $\left[{ }^{\circ}\right]$ & Relative error $[\%]$ \\
\hline 10 & 13 & 3 & $30 \%$ \\
30 & 35 & 5 & $16.7 \%$ \\
60 & 63 & 3 & $5 \%$ \\
90 & 91 & 1 & $1.1 \%$ \\
120 & 120 & 0 & $0 \%$ \\
150 & 152 & 2 & $1.3 \%$ \\
180 & 180 & 0 & $0 \%$ \\
210 & 212 & 2 & $0.95 \%$ \\
240 & 246 & 6 & $2.5 \%$ \\
270 & 273 & 3 & $1.1 \%$ \\
300 & 302 & 2 & $0.67 \%$ \\
330 & 330 & 0 & $0 \%$ \\
360 & 360 & 0 & $0 \%$ \\
\hline
\end{tabular}


Table 2 Analyze the rotation angle error under different frequency division parameters

\begin{tabular}{ccccc}
\hline $\begin{array}{c}\text { Frequency division } \\
\text { parameters }\end{array}$ & $\begin{array}{c}\text { Rotation } \\
\text { angle }\left[{ }^{\circ}\right]\end{array}$ & $\begin{array}{c}\text { Actual rotation } \\
\text { angle }\left[{ }^{\circ}\right]\end{array}$ & Error $\left[{ }^{\circ}\right]$ & Relative error [\%] \\
\hline 1 & 90 & 91 & 1 & $1.1 \%$ \\
2 & 90 & 92.5 & 2.5 & $2.70 \%$ \\
4 & 90 & 91 & 1 & $1.10 \%$ \\
8 & 90 & 93 & 3 & $3.30 \%$ \\
16 & 90 & 90 & 0 & $0 \%$ \\
\hline
\end{tabular}

\section{Conclusions}

By design the program and control the corresponding hardware devices, to design a Stepping motor control system to control the rotation of the stepping motor. The software part of this system is programmed based on LabVIEW, and communicates with the ELVIS II platform via USB port of the computer, so as to carry out a series of control on stepping motor. The hardware part consists of four parts, including the computer, the NI ELVIS II platform, the drive and the stepping motor. Through the LabVIEW program in the computer input the rotation angle. The rotation angle information is transmitted from the computer hardware interface to the NI ELVIS II platform and generates corresponding electrical signal. The electrical signal is transmitted to the driver and drives the Stepping motor to perform the corresponding rotation angle and control the rotation direction. The system test shows that the rotation angle is larger, the actual rotation error is smaller, and the frequency coefficient of stepping motor is larger, the actual rotation error is smaller. In addition to controlling the stepping motor, this system could be used in the production line and machine processing operation control.

\section{Acknowledgements}

This work was financially supported by the Educational Research Fund of Hainan Province Education Department (Grant No. Hnjg2016ZD-20), the National Student Innovation and Entrepreneurship Training Program(Grant No. 201613892119, 201713892009) and Hai Nan Student Innovation and Entrepreneurship Training Program(Grant No. 20160257, 20160202).

\section{References}

[1] H. Qiu, W. Lin and S. Z. Huang: Chinese Journal of Electron Devices (2011), Vol.34, No.6, p. 686

[2] L. Z. Sun: The Design of the Stepping Motro PC Remote Control System, Da Qing: Northeast Petroleum University (2010).

[3] F. Liu, W. H. Yang and S. Xie: Information Technology (2013), Vol.37, No.9, p. 10

[4] J. Q. Mao, N. Z. Yun, Y. Meng and N. S. Qu: Electric Machines \& Control Application (2009), Vol.36, No.1, p.30

[5] S. Q. Zhao, X. D. Jing, F. Q. Qi and Y. Zhang: Coal Mine Machinery (2012), Vol.33, No.6, p.79

[6] C. Tian, C. Su: Electronic Test (2017), No.22, p.5

[7] K. Huang, X. J. Hao: Electronic Design Engineering(2009), Vol.17, No.1, p.72 